\title{
ESTIMATION OF VITAMIN D GINGIVAL CREVICULAR FLUID LEVEL IN PATIENTS WITH DIFFERENT PERIODONTAL CONDITIONS
}

\author{
Ahmed El Barbary* and Marwa Hegab ${ }^{* *}$
}

\begin{abstract}
Background: Vitamin D (Vit. D) is responsible in the process of calcium homeostasis, among many other biological effects. Thus it plays an essential role in bone related diseases. Furthermore, several studies investigated the link between Vit. D plasma level and various conditions of periodontal diseases.
\end{abstract}

Objective: Therefore, the present study aimed to assess Vit. D3 [25(OH) D] gingival crevicular fluid (GCF) levels in individuals with different periodontal conditions.

Subjects and methods: $60 \mathrm{GCF}$ samples were taken from 60 individuals divided into 3 equal groups, healthy, gingivitis and periodontitis. Vit. D GCF level was assessed using ELISA technique.

Results: There was a statistically significant difference between (gingivitis, periodontitis and healthy control) groups, with mean and SD $[4.4 \pm 0.3 \mathrm{ng} / \mathrm{ml}, 3.5 \pm 0.4 \mathrm{ng} / \mathrm{ml}, 5.6 \pm 0.5 \mathrm{ng} / \mathrm{ml}]$ respectively (P value 0.001).

Conclusion: The results are supporting the link between $25(\mathrm{OH})$ D GCF level and the periodontal condition. The study might be emphasizing the correlation between the periodontal disease and deficiency in Vit. D.

KEYWORDS: Gingival crevicular fluid, gingivitis, periodontitis, periodontal disease, Vitamin D

\section{INTRODUCTION}

Periodontal diseases are a group of complex diseases caused by microbial biofilms and if not treated, cause gradual destruction of connective tissue, alveolar bone and finally lead to tooth mobility and loss. Periodontitis is considered a highly prevalent oral disease affecting up to $50 \%$ of adults over 30 years and $60 \%$ of those over 65 years of age (Schultz et al, 2015). Several risk factors may modify the severity of periodontal disease and its destruction rate. These factors comprise patient's age, systemic diseases, habits, and genetic background (Van Dyke \& Dave, 2005).

* Assistant professor Oral Medicine and Periodontology Department, Faculty of Dentistry, Cairo University

** Lecturer, Oral Medicine and Periodontology Department, Faculty of Dentistry, Cairo University 
The fat soluble Vit. D is having 2 forms, the first is 25-hydroxy Vit. D [25(OH) D] which has valuable biological functions. $25(\mathrm{OH}) \mathrm{D}$ is activated into the second form, 1,25-hydroxy Vit. D [1,25(OH) D], which offers most of the biological actions that include the regulation of homeostasis of the nervous, musculoskeletal, cardiovascular systems among others (Holick 2008). Vit. D deficiency is usually recognized as a blood level of [25(OH) D] below $20 \mathrm{nmol} / \mathrm{L}$ (Cashman, 2015).

Vit. D has been identified as an anabolic factor, and Vit. D systemic administration helps in prevention of bone destruction occurring in osteoporosis. Moreover, it is clear that it may boost bone formation in bony-related disorders (Liu et al., 2009 ; Lou et al., 2013). Furthermore, Vit. D has, antiinflammatory, antimicrobial and antineoplastic actions. (Khammissa et al., 2018). Moreover, Vit. D receptors expression on surfaces of certain immune cells suggests that such cells are responsive to Vit. D and indicates that vit. D has a vital effect in regulating the immune response (Prietel et al., 2013). Wang et al., (2004) and Hertting et al., (2010) showed that Vit. D was found to improve innate immunity by enhancing expression of antimicrobial peptides.

Vit. D also plays an anti-inflammatory role as stated by Zhang et al., (2012), the authors showed that Vit. D attenuates inflammation by suppressing release of some inflammatory cytokines such as interleukin (IL6) and tumor necrosis factor alpha (TNFa) production (Griffin et al., 2003 ; Aranow 2011). There has been a lot of concern in discovering serum Vit. D deficiency impact in inflammatory diseases such as rheumatoid arthritis, tuberculosis and periodontal disease (Anbarcioglu et al., 2018). Multiple clinical trials investigated the relation of Vit. D with the pathogenesis of periodontitis and this point has become of a great interest to confirm or deny such relationship (Liu et al., 2009 ; Karasneh et al., 2013 ; Aberu et al., 2016 ; Meghil et al.,2019).

This correlation between Vit. D and periodontal disease was based on that Vit. D induces cathelicidin which is an antimicrobial protein that helps in immune reaction in periodontal tissues. Human gingival and periodontal ligament fibroblasts has shown to be producing Vit. D which leads to cathelicidin activation. Serum $25(\mathrm{OH}) \mathrm{D}$ deficiency is accompanied by suppressed levels of cathelicidin levels in tissues of many patients suffering from periodontal diseases (Zhou et al., 2018 ; Bayirili et al., 2020).

Moreover, Grenier et al., (2016) had pointed that $1,25(\mathrm{OH}) \mathrm{D}$ suppresses the gene expression of the virulence factors of some periodontal pathogens. They also highlighted that 1,25 $(\mathrm{OH}) \mathrm{D}$ suppresses the nuclear factor kappa (NF-kB) activation in the inflammatory cells leading to a decrease of proinflammatory cytokines production. Gao et al., (2020) and Khan \& AbdulAhad (2021) mentioned that the systemic administration of Vit. D as an adjunct to non surgical periodontal therapy led to significant improvement in terms of decrease in pocket depth and clinical attachment gain.

Furthermore, several studies investigated and confirmed the correlation between Vit. D plasma level and the different periodontal diseases (Millen et al., 2013; Jimenez et al. 2014; Antonoglou et al., 2015; Lee et al., 2015; Anbarcioglu et al., 2018; Peric et al., 2018 ; Isola et al., 2019). However, very few studies assessed Vit. D level in GCF and its relation with the periodontal condition. Therefore, the purpose of the current study was to evaluate Vit. D GCF level in patients with different periodontal conditions.

\section{SUBJECTS AND METHODS}

This study was conducted at the Department of Diagnosis, Oral medicine, and Periodontology, Faculty of dentistry, Cairo University by screening patients to select the required sample. Sixty adults, aged 31-60 years, were included, and equally divided into three groups; healthy as control, gingivitis and periodontitis Grade A and B (Caton et al., 2018). Epi-info $^{\mathrm{TM}}$ software, a statistical software for 
epidemiology, was used for sample size calculation. The acceptable margin of error was set to be $5 \%$ (0.05). The expected total sample size was 60 , with a confidence level of $80 \%$.

Any patient with any systemic conditions that might affect or modify the periodontal condition were excluded from the study. Exclusion criteria also included smoking and pregnancy. The study protocol complied with the guidelines of the Helsinki Declaration, all the steps of this study were explained in full details to every participating subject who signed an informed consent. After taking a proper full medical and dental history, oral examination was performed. Plaque index (PI), gingival index (GI) (Löe 1967) and percentage of bleeding on probing (BOP) were recorded. Clinical attachment loss (CAL) and probing depth (PD) were measured at 6 sites per each selected tooth (mesial, mid, distal of buccal and lingual/palatal aspects) using the periodontal probe with Williams markings (Shaswata and Shobha 2019). These measurements were approximated to the nearest millimeter (Chrysanthakopoulos, 2014; Wagner et al., 2016). Gingivitis and periodontitis patients received phase 1 periodontal therapy. This consisted of full mouth ultrasonic and manual supra, subgingival scaling and root planing (for periodontitis group) after collection of GCF samples.

After the determination of the sites for GCF sampling, proper dryness was performed followed by careful isolation of these sites using cotton rolls to avoid saliva contamination. A filter paper $(2 \mathrm{X}$ $9 \mathrm{~mm}$ ) was inserted into the sulcus or pocket until mild resistance was detected. The paper was left for 30 seconds to allow for the collection of an appropriate amount of GCF. Afterwards, the paper was withdrawn and placed in a plastic Eppendorf tube and then kept frozen at $-20{ }^{\circ} \mathrm{C}$ until assayed. Any paper with any blood contamination was discarded to avoid any errors in assessment of Vit. $\mathrm{D}$ level, and the sampling procedure was performed again 30 minutes later (Yuce et al., 2017).
Vit.D3 level was quantified in GCF samples of all patients using Human 25-Dihydroxy Vit. D (25-OH -D) enzyme-linked immunosorbent assay (ELISA) kit (Sunred bio, Shanghai, China). This kit is based on the double antibody sandwich (ELISA) principle for assessing the level of Human 25-Dihydroxy Vit. D in body fluid samples. 25-Dihydroxy Vit. D (25-OH-D) was added to monoclonal antibody enzyme well pre-coated with Human 25-Dihydroxy Vit. D. According to the manufacturer instructions, incubation was done; then, (25-OH-D) antibodies marked with biotin was added, and linked with Streptavidin-HRP to create an immune complex; then incubation and washing were performed once more to remove any uncombined enzyme. Then A, $\mathrm{B}$ chromogens were added and the solution color was converted into blue. The concentrations of human substance (25-OH-D) were then positively correlated with color intensity.

\section{Statistical analysis}

Categorical data were analyzed using the Chi square test, numerical data were presented as mean and standard deviation (SD). Parametric data were analyzed using one way repeated measures ANOVA followed by Bonferroni post hoc test. Non parametric data were presented as median and range and analyzed using Kruskal-Wallis test followed by Mann-Whitney test. P-value $<0.05$ was considered as statistical significance. The Statistics Package for Social Sciences (SPSS) software version 25 was used for the statistical analysis.

\section{RESULTS}

GCF samples were collected from 60 adult patients attending the clinic of periodontology, Oral Medicine, diagnosis and Periodontology department, Faculty of dentistry, Cairo University. The 60 patients were distributed into 3 groups (20 patients in each), healthy gingiva (control), gingivitis and periodontitis. As shown in table (1), there is no statistical significant difference between all groups concerning age and gender $\left(\begin{array}{ll}P & 0.064)\end{array}\right)\left(\begin{array}{l}P \\ 0.159\end{array}\right)$ 
respectively. There is also a statistical significant difference between the control group and (gingivitis and periodontitis) groups in plaque index (PI), gingival index (GI), percentage of bleeding on probing (BOP) $(P<0.001)$. While, there is a statistical significant difference between the (control/ gingivitis) groups and periodontitis groups in pocket probing depth and clinical attachment loss $(P<0.001)$.

Data related to Vit. D3 GCF levels are presented in tables $(2 \& 3)$. The results show a statistically significant difference between all groups in Vit. D3 level with mean, SD and (median) $[4.4 \pm 0.3$ $\mathrm{ng} / \mathrm{ml}(4.3) ; 3.5 \pm 0.4 \mathrm{ng} / \mathrm{ml}(3.4) ; 5.6 \pm 0.5 \mathrm{ng} / \mathrm{ml}$ $(5,7)]$ in gingivitis, periodontitis and healthy control groups respectively $(P \quad 0.001)$ Vit. D3 level was statistically significantly higher in healthy group in comparison to both diseased groups ( $P$ 0.000). In gingivitis group, Vit. D3 level is statistically significantly greater than in periodontitis group $(P$ 0.001) as shown in fig. (1).

TABLE (1) Mean and SD of clinical parameters in the study groups

\begin{tabular}{ccccc}
\hline Clinical Parameters & Control group & Gingivitis group & Periodontitis group & P-value \\
\hline 1- P.I. & $0.29+0.08^{\mathrm{a}}$ & $1.42+0.27^{\mathrm{b}}$ & $1.53+0.30^{\mathrm{b}}$ & $<0.001^{*}$ \\
\hline 2- G.I. & $0.44+0.18^{\mathrm{a}}$ & $1.40+0.32^{\mathrm{b}}$ & $1.58+0.32^{\mathrm{b}}$ & $<0.001^{*}$ \\
\hline 3- B.O.P. \% & $5.18+1.03^{\mathrm{a}}$ & $75.5+21.41^{\mathrm{b}}$ & $84.5+12.4^{\mathrm{b}}$ & $<0.001^{*}$ \\
\hline 4- P.P.D. & $2.19+0.18^{\mathrm{a}}$ & $3.18+0.13^{\mathrm{a}}$ & $5.5+0.88^{\mathrm{b}}$ & $<0.001^{*}$ \\
\hline 5- C.A.L. & $0.00+0.00^{\mathrm{a}}$ & $0.00+0.00^{\mathrm{a}}$ & $5.61+1.01^{\mathrm{b}}$ & $<0.001^{*}$ \\
\hline 6- Age & $44.93 \pm 13.8$ & $42.87 \pm 11.2$ & $49.43 \pm 10.7$ & 0.064 \\
\hline 7- Gender (Male \%) & $39 \%$ & $45 \%$ & $46 \%$ & 0.159 \\
\hline
\end{tabular}

Different letters indicate statistical significant difference $(p<0.05)$.

TABLE (2) Mean, SD and median of Vit. D GCF levels in the study groups

\begin{tabular}{ccccc}
\hline Group & Mean \pm Standard Deviation $(\mathbf{n g} / \mathbf{m l})$ & Median $(\mathbf{I Q R})$ & Kruskal Wallis Test & P \\
\hline Gingivitis & $4.4 \pm 0.3^{\mathrm{a}}$ & $4.3(4.1-4.6)$ & & \multirow{2}{*}{22.207} \\
\cline { 1 - 3 } Periodontitis & $3.5 \pm 0.4^{\mathrm{b}}$ & $3.4(3.2-3.6)$ & & $0.000^{*}$ \\
\cline { 1 - 3 } Control & $5.6 \pm 0.5^{\mathrm{c}}$ & $5.7(5.2-6.0)$ & & \\
\hline
\end{tabular}

Different letters indicate statistical significant difference $(p<0.05)$.

TABLE (3) Comparison between the Vit. D GCF levels in the three study groups

\begin{tabular}{ccc}
\hline Comparison between & $\begin{array}{c}\text { Mann-Whitney } \\
\text { U test }\end{array}$ & $\mathbf{P}$ \\
\hline Gingivitis \& Periodontitis & 2.500 & $0.001^{*}$ \\
\hline Gingivitis \& Control & 1.000 & $.000^{*}$ \\
\hline Periodontitis \& Control & .000 & $.000^{*}$ \\
\hline
\end{tabular}

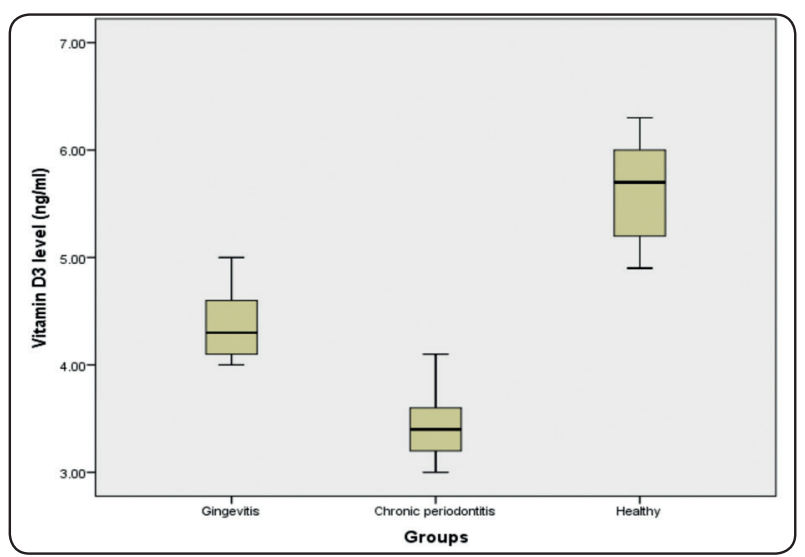

Fig. (1) Showing comparison of D3 GCF levels in the different study groups 


\section{DISCUSSION}

The information obtained from epidemiological studies is hard to get from the other study designs. In periodontology, the information obtained from epidemiological studies usually includes the prevalence of different periodontal conditions among a given population, and the possible relationship between the numerous etiologic and/or risk factors and the progression of such conditions (Tugnait and Clerehugh, 2001; Eke et al., 2012).

Some studies have confirmed the effect of Vit. D3 in protection against inflammation and chronic diseases including the periodontal disease (Guillot et al., 2010 ; Tang et al., 2013). In addition, Vit. D deficiency (Anbarcioglu et al., 2019), increased levels of plasma proteins that bind to Vit. D (Zhang et $a l ., 2013)$ and Vit. D receptors gene polymorphism (Park et al., 2006 ; Karasneh 2013 ; Guo et al., 2019), have been strongly linked with aggressive periodontitis.

Many studies investigated the relation between Vit. D levels and periodontal diseases, most of these studies were performed using patients serum samples (Hiremath et al., 2012 ; Eshghi et al., 2016 ; Yoon et al.,2017). 25(OH) D was the most commonly form used to assess Vit. D level, due its long life in body fluids reaching 20 days, on the other side $1,25(\mathrm{OH}) \mathrm{D}$ occurs only for up to 4 hours (Madi et al., 2020).

The majority of the studies confirmed the indirect proportional relation between serum Vit. D level and the periodontal disease. They found that the serum level decreased with periodontitis when compared to periodontal health condition (Dietrich et al., 2004 and 2005 ; Amano 2009 ; Boggess 2011 and Hiremath et al., 2012 ; yoon et al., 2017). On the other side, Bonnet et al., 2019 provided modest evidence for such correlation and other studies failed completely to prove any association. (Garcia et al., 2011 ; Antonoglou et al., 2015a; Eshghi et al., 2016). Peric et al., 2018 concluded that the proof of the impact of Vit. D levels on the periodontal condition and whether it is a predisposing factor or a consequence of the disease remains scarce and controversial. Therefore, the current study was aiming to assess and compare $25(\mathrm{OH}) \mathrm{D}$ GCF levels in individuals with periodontal diseases (gingivitis, periodontitis) versus those with healthy periodontium (as control).

This study's results have reported that Vit. D levels in GCF can be related to the periodontal condition. The highest level was identified in samples collected from patients with healthy periodontium, followed by those with gingivitis. lastly, the samples collected from the periodontitis patients showed the lowest GCF levels of Vit. D, indicating an inverse proportional relationship between the severity of periodontal disease and GCF Vit. D level.

These outcomes are in accordance with the results reported by Matoug et al 2014 who found a statistical significant difference between Vit. D GCF levels in individuals with periodontitis and gingivitis with mean (3.41 vs $4.86 \mathrm{ng} / \mathrm{mL}$ respectively) in favor of the latter. Moreover, Lary et al., (2016) found that deficient 25(OH) D levels were detected in $48.3 \%$ of subjects with advanced periodontitis versus only $13.8 \%$ in the control group. They stated that $25(\mathrm{OH}) \mathrm{D}$ serum levels are significantly related with the periodontal disease incidence and rate of progression. This association indicates that, may be due to its immuno-modulatory role, antiinflammatory properties, and antimicrobial action, an acceptable serum level of Vit. D is essential for the maintaining the periodontal health. (Khammissa et al., 2018)

Furthermore, Bahargava (2019) recorded a statistically significant interrelation between serum $25(\mathrm{OH}) \mathrm{D}$ level and the parameters such as GI, PPD and CAL highlighting an overall low levels in periodontitis patients with no decrease in levels with the increase in disease severity. Similarly, Isola et al., (2019) reported a significantly lower 
Vit. D serum levels in individuals suffering from periodontitis alone or in conjunction with ischemic heart disease when compared to individuals suffering from only ischemic heart disease or healthy controls. Moreover, Madi et al., (2020) stated that serum Vit. D level had been considered a crucial factor which might reflect and influence the periodontal condition. They recorded a mean of alveolar crest height of $1.6 \mathrm{~mm}$ in patients with no Vit. D deficiency, a mean of $3.1 \mathrm{~mm}$ when Vit. D level was less than $20 \mathrm{ng} / \mathrm{mL}$, and $4.6 \mathrm{~mm}$ when the level was less $10 \mathrm{ng} / \mathrm{mL}$.

On the contrary, in a previous study performed by Antonoglou et al., (2015), a greater 25(OH) D serum level in periodontally healthy patients was recorded when compared to periodontitis patients (56.9 vs $41.9 \mathrm{~nm} / \mathrm{L}$ ) respectively. However, they found no statistical significant difference between both groups and could not prove any true correlation between serum 25(OH) D concentration and periodontal health. On the other hand, they reported a statistically significant difference between 1,25 $(\mathrm{OH}) \mathrm{D}$ serum concentrations in periodontitis and periodontally healthy subjects with mean of (77 $\mathrm{pmol} / \mathrm{L}$ vs $106 \mathrm{pmol} / \mathrm{L}$ ) respectively in favor of the periodontally healthy patients. They attributed this to the lower serum level of $25(\mathrm{OH}) \mathrm{D}$ in $93 \%$ of the selected patients of both groups in their study.

Furthermore, Lee et al., (2015) also reported a significant interrelationship between Vit. D deficiency and the periodontal condition in smokers group but not in non smokers. Similarly, Lee et al., (2021) failed to show any significant interrelation between Vit. D plasma concentrations and periodontal condition, reporting no statistical significant difference in periodontitis prevalence between patients with average versus deficient $25(\mathrm{OH}) \mathrm{D}$ levels. In their opinion, this could be justified by the use of community periodontal index (CPI) as a measure for assessing the periodontal condition, which is different than the use of gingival index, CAL and PD in diagnosis of periodontal disease and its severity. Hence, the inclusion of representative teeth and gingival pockets must have affected the accuracy in estimation of periodontitis prevalence in their study.

Constantini et al., (2020) detected a higher level of salivary Vit. D in subjects with periodontal health with a median of $(4.6 \mathrm{ng} / \mathrm{mL})$ than that in periodontits patients with a median of $(3.7 \mathrm{ng} /$ $\mathrm{mL})$. However, this difference was statistically insignificant, this disparity in results might be related to the use of salivary samples in their study to assess Vit. D levels.

Finally, the results of this study reported that Vit. $\mathrm{D}$ deficiency might be associated with a greater risk for periodontal disease. This is strongly supported by the systematic reviews conducted by Pinto et al., (2018) and Machado et al., (2020) who confirmed the possibility of considering decreased serum Vit. D levels a potential risk factor for periodontal disorders. However, due to the limitations and the equivocal findings of the included studies, it was impossible to confirm the relationship between periodontal disorders and levels of Vit. D.

\section{CONCLUSIONS}

Within the current study's limitations, this study supports the link between 25(OH) D GCF levels and the periodontal condition. Patients diagnosed with periodontitis showed lowest GCF levels of $25(\mathrm{OH}) \mathrm{D}$ followed by those with gingivitis. The highest levels were in periodontally healthy patients. However, additional studies with larger sample size with subgrouping of different periodontitis stages are needed to further realize the link of Vit. D levels especially in GCF and periodontal disease.

\section{Conflict of interest and funding}

There was no conflict of interest. This study was self-funded. 


\section{REFERENCES}

1. Abreu, O. J., Tatakis , D.N., Elias - Boneta, A.R., Del Valle L.L., Hernandez R., Pousa M.S., \& Palacios C. Low Vit. D status strongly associated with periodontitis in Puerto Rican adults. BMC Oral Health, 2016, 16 (1), 89.

2. Amano Y, Komiyama K, Makishima M. Vit. D and periodontal disease. J Oral Sci 2009;51:11-20.

3. Anbarcioglu E, Kirtiloglu T, Öztürk A, Kolbakir F, Acıkgöz $\mathrm{G}$, Colak R. Vit. D deficiency in patients with aggressive periodontitis. Oral Dis. 2019;25:242-249.

4. Antonoglou GN, Knuuttila M, Niemel ostalo P, Raunio T, Hiltunen L, Karttunen R, Tervonen T. Serum parathyroid hormone and active Vit. D in chronic periodontitis. J Clin Periodontol 2015; 42: 726-732.

5. Antonoglou GN, Knuuttila M, Niemel, O, Raunio T, Karttunen R, Vainio O, Hedberg P, Ylostalo P, Tervonen T. Low serum level of $1,25(\mathrm{OH}) \mathrm{D}$ is associated with chronic periodontitis. J Periodont Res 2015; 50: 274-280.

6. Aranow, C. Vit. D and the immune system. Journal of Investigative Medicine, 2011, 59(6), 881-886.

7. Bayirli B.A., Öztürk A., Avci B. Serum Vit. D concentration is associated with antimicrobial peptide level in periodontal diseases. Arch. Oral Biol. 2020;117:104827.

8. Bhargava A, Rastogi P, Lal N, Singhal R, Khatoon S, Ali Mahdi A. Relationship between VIT. D and chronic periodontitis. J Oral Biol Craniofac Res. 2019 Apr-Jun; 9(2):177-179.

9. Boggess, K.A., Espinola, J.A., Moss, K., Beck, J., Offenbacher, S., Camargo Jr, C.A. Vit. D status and periodontal disease among pregnant women. J. Periodontol., 2011, 82, 195-200

10. Bonnet, C., Rabbani, R., Moffatt, M.E.K., Kelekis-Cholakis, A., Schroth, R.J. The Relation Between Periodontal Disease and Vit. D. J. Can Dent., 2019, Assoc. 85, 1488-2159

11. Cashman K.D., "Vit. D: dietary requirements and food fortification as a means of helping achieve adequate Vit. D status," Journal of Steroid Biochemistry and Molecular Biology, 2015, vol. 148, pp. 19-26.

12. Caton. J, Armitage. G, Berglundh. T, et al. A new classification scheme for periodontal and perimplant diseases and conditions: Introduction and key Changes from the 1999 classification. J Clin Periodontol. 2018; 45(Suppl 20)

13. Chrysanthakopoulos, N. A. 'Aetiology and severity of gingival recession in an adult population sample in Greece.', Dental research journal., 2011; 8(2), pp. 64-70.
14. Chrysanthakopoulos NA. Gingival recession: Prevalence and risk indicators among young greek adults. J Clin Exp Dent. 2014;6(3):e243-9

15. Costantini E, Sinjari B, Piscopo F, et al. Evaluation of Salivary Cytokines and Vit. D Levels in Periodontopathic Patients. Int J Mol Sci. 2020;21(8):2669.

16. Dietrich T, Joshipura KJ, Dawson-Hughes B, BischoffFerrari HA. Association between serum concentrations of 25-hydroxyVit. D and periodontal disease in the US population. Am J Clin Nutr 2004;80:108-13.

17. Dietrich T, Nunn M, Dawson-Hughes B, BischoffFerrari HA. Association between serum concentrations of 25-hydroxyVit. D and gingival inflammation. Am J Clin Nutr $2005 ; 82: 575-80$

18. Eke P, Thornton-Evans G, Dye B, and Genco R (2012): Advances in Surveillance of Periodontitis: The Centers for Disease Control and Prevention Periodontal Disease Surveillance. Project J Periodontol 1337-1342.

19. Gao W, Tang H, Wang D, Zhou X, Song Y, Wang Z. Effect of short-term Vit. D supplementation after nonsurgical periodontal treatment: A randomized, double-masked, placebo-controlled clinical trial. J Periodontal Res. 2020 Jun; 55(3):354-362.

20. Garcia, M.N., Hildebolt, C.F., Miley, D.D., Dixon, D.A., Couture, R. A., Anderson Spearie, C.L., Langenwalter, E.M., Shannon, W.D., Deych, E., Mueller, C.. One-year effects of Vit. D and calcium supplementation on chronic periodontitis. J. Periodontol. 2011, 82, 25-32

21. Grenier D, Morin MP, Fournier-Larente J, Chen H. Vit. D inhibits the growth of and virulence factor gene expression by Porphyromonas gingivalis and blocks activation of the nuclear factor kappa B transcription factor in monocytes. J Periodontal Res 2016;51:359-65

22. Griffin, M.D., Xing, N., \& Kumar, R. Vit.D and its analogs as regulators of immune activation and antigen presentation. Annual Review of Nutrition, 2003, 23(1), 117-145.

23. Guillot X, Semerano L, Saidenberg-Kermanac'h N, Falgarone G, Boissier MC: Vit. D and inflammation. Joint Bone Spine, 2010, 77, 552-557.

24. Guo H-X, Pan J, Pan H-B, Cui S-J, Fang C-Y. Correlation of Vit. D receptor gene (ApaI) polymorphism with periodontitis: A meta-analysis of Chinese population. Food Sci Nutr. 2019;7:3607-3612. 
25. Hertting, O., Holm, Å., Lüthje, P., Brauner, H., Dyrdak, R., Jonasson, A. F., Brauner, A. Vit. D induction of the human antimicrobial Peptide cathelicidin in the urinary bladder. PLoS ONE, 2010, 5(12), e15580.

26. Hiremath VP, Rao CB, Naik V, Prasad KV. Multivariate analysis of association of serum Vit. D levels of $25(\mathrm{OH})$ D with marginal gingivitis. Int J Med Public Health. 2012;2:50-55.

27. Holick MF. The Vit. D deficiency pandemic and consequences for nonskeletal health: Mechanisms of action. Mol Aspects Med 2008;29:361-8.

28. Isola G, Alibrandi A, Rapisarda E, Matarese G, Williams RC, Leonardi R. Association of Vit. D in patients with periodontitis: A cross-sectional study. J Periodont Res. 2020;55:602-612.

29. Karasneh JA, Ababneh KT, Taha AH, Al-Abbadi MS, Marzouka NS, Jaradat SM, Thornhill MH. Association of Vit. D receptor gene polymorphisms with chronic and aggressive periodontitis in Jordanian patients. Eur J Oral Sci 2013; 121: 551-558.

30. Khammissa RAG, Ballyram R, Jadwat Y, Fourie J, Lemmer J, and Feller L: Vit. D Deficiency as It Relates to Oral Immunity and Chronic Periodontitis. International Journal of Dentistry, 2018 1-9.

31. Khan Saif , Abdul Ahad (2021) Application of adjunct Vit. D supplementation in the management of periodontal disease: A three-pronged approach. Journal of Dental Sciences, 2021, 16(1):534-535

32. Kingman A, Albandar JM. Methodological aspects of epidemiological studies of periodontal diseases. Periodontol 2000, 2002;29:11-30.

33. Jimenez, M., Giovannucci, E., Kaye, E. K., Joshipura, K. J., \& Dietrich, T. (2014). Predicted Vit. D status and incidence of tooth loss and periodontitis. Public Health Nutrition, 17(4), 844-852.

34. Lee H-J, Je D-I, Won S-J, Paik D-I, Bae K-H. Association between Vit. D deficiency and periodontal status in current smokers. Community Dent Oral Epidemiol 2015; 43: 471-478.

35. Lee, M.-R.; Han, S.-J.; Kim, H.-E.; Choi, J.-S. Relationship between Vit. D Deficiency and Periodontitis in Korean Adults Aged $\geq 60$ Years: Analysis of Data from the Korea National Health and Nutrition Examination Survey (2013-2014). Int. J. Environ. Res. Public Health 2021, 18, 4181.
36. Liu K, Meng H, Hou J (2012) Activity of 25-hydroxylase in human gingival fibroblasts and periodontal ligament cells. PloS One, 2012;7(12): e52053

37. Liu, K., Meng, H., Tang, X., Xu, L., Zhang, L., Chen, Z., Lu, R. (2009). Elevated plasma calcifediol is associated with aggressive periodontitis. Journal of Periodontology, 80( 7), 1114-1120.

38. Luo J, Wen H, Guo H, Cai Q, Li S, Li X: 1,25-dihydroxyVit. D3 inhibits the RANKL pathway and impacts on the production of pathway-associated cytokines in early rheumatoid arthritis. Biomed Res Int, 2013101805.

39. Machado V, Lobo S, Proença L, Mendes JJ, Botelho J. Vit. D and Periodontitis: A Systematic Review and Meta-Analysis. Nutrients. 2020;12(8):2177..

40. Madi M., Pavlic V., Alammar S.M., Alsulaimi L.M., Alotaibi R.S., AlOtaibi G.M., Zakaria O., The association between Vit. D level and periodontal disease in Saudi population, a preliminary study, The Saudi Dental Journal, 2020, ISSN 1013-9052,

41. Meghil MM, Hutchens L, Raed A, et al. The influence of Vit. D supplementation on local and systemic inflammatory markers in periodontitis patients: A pilot study. Oral Dis. 2019;25:1403-1413.

42. Millen, A. E., Hovey, K. M., LaMonte, M. J., Swanson, M., Andrews, C. A., Kluczynski, M. A., Wactawski-Wende, J. (2013). Plasma 25- hydroxyVit. D concentrations and periodontal disease in post menopausal women. Journal of Periodontology, 84(9), 1243-1256.

43. Park KS, Nam JH, Choi J. The short Vit. D receptor is associated with increased risk for generalized aggressive periodontitis. J Clin Periodontol 2006; 33: 524-528.

44. Perić M, Cavalier E, Toma S, Lasserre JF. Serum Vit. D levels and chronic periodontitis in adult, Caucasian population - a systematic review. J Periodont Res. 2018;53:645-656.

45. Pinto, J., Goergen, J., Muniz, F., Haas, A.N.: Vit. D levels and risk for periodontal disease: A systematic review. J. Periodontal Res. 2018 53, 298-305

46. Prietl B, Treiber G, Pieber TR, Amrein K. Vit. D and immune function. Nutrients. $2013 \mathrm{Jul}$ 5;5(7):2502-21.

47. Schulze-Spate U., Turner R, Wang Y. et al., "Relationship of bone metabolism biomarkers and periodontal disease: the osteoporotic fractures in men (MrOS) study," Journal of Clinical Endocrinology \& Metabolism, 2015 vol. 100, $6,2425-2433$ 
48. Tang X, Pan Y, Zhao Y (2013) Vit. D inhibits the expression of interleukin-8 in human periodontal ligament cells stimulated with Porphyromonas gingivalis. Arch Oral Biol 58, 397-407.

49. Tugnait, A. and Clerehugh, V.: 'Gingival recession-its significance and management.', Journal of dentistry, 2001, 29(6), 381-94.

50. Van Dyke, T.E., \& Dave, S. Risk factors for periodontitis. Journal of the International Academy of Periodontology, 2005, 7(1), 3 .

51. Wang, T.-T., Nestel, F. P., Bourdeau, V., Nagai, Y., Wang, Q., Liao, J., Mader, S. Cutting edge: 1, 25-dihydroxyVit. D3 is a direct inducer of antimicrobial peptide gene expression. The Journal of Immunology, 2004 173(5), 2909-2912.

52. Yoon, Na-Na \& Lee, Ji-Young \& Yu, Byeng-Chul. (2017). Association between Vit. D Level in Blood and Periodontitis in Korean Elderly. Journal of Dental Hygiene Science., 2017; 17, 233-241.
53. Yuce HB, Gokturk O, Turkal HA, Inanir A, Benli I, and Demir O: Assessment of local and systemic 25-hydroxyVit. D, RANKL, OPG, and TNF levels in patients with rheumatoid arthritis and periodontitis Journal of Oral Science, 2017, Vol. 59, No. 3, 397-404.

54. Zhang, Y., Leung, D. Y., Richers, B. N., Liu, Y., Remigio, L. K., Riches, D.W., \& Goleva, E. Vit. D inhibits monocyte/macrophage proinflammatory cytokine production by targeting MAPK phosphatase-1. The Journal of Immunology, 2012; 188(5), 2127-2135.

55. Zhang X, Meng H, Sun X, Xu L, Zhang L, Shi D, Feng X, Lu R, Chen Z. Elevation of Vit. D-binding protein levels in the plasma of patients with generalized aggressive periodontitis. J Periodont Res 2013; 48: 74-79. _ 2012 John Wiley \& Sons A/S

56. Zhou X., Zhang P., Wang Q., Xia S., Ji N., Ding Y., Wang Q. 25-hydroxyVit. D3 alleviates experimental periodontitis via promoting expression of cathelicidin in mice with type 2 diabetic mellitus. J. Nutr. Sci. Vitaminol. (Tokyo) 2018;64:307-315. 